\title{
Cosmic censorship in overcharging a Reissner-Nordström black hole via charged particle absorption
}

\author{
Soichiro Isoyama * Norichika Sago $\oplus$ and Takahiro Tanak团 \\ Yukawa Institute for Theoretical Physics, Kyoto university, Kyoto, 606-8502, Japan
}

(Dated: July 14, 2018)

\begin{abstract}
There is a claim that a static charged black hole (Reissner-Nordström black hole) can be overcharged by absorbing a charged test particle. If it is true, it might give a counter example to the weak cosmic censorship conjecture, which states that spacetime singularities are never observed by a distant observer. However, so far the proposed process has only been analyzed within a test particle approximation. Here we claim that the back reaction effects of a charged particle cannot be neglected when judging whether the suggested process is really a counter example to the cosmic censorship conjecture or not. Furthermore, we argue that all the back reaction effects can be properly taken into account when we consider the trajectory of a particle on the border between the plunge and bounce orbits. In such marginal cases we find that the Reissner-Nordström black hole can never be overcharged via the absorption of a charged particle. Since all the plunge orbits are expected to have a higher energy than the marginal orbit, we conclude that there is no supporting evidence that indicates the violation of the cosmic censorship in the proposed overcharging process.
\end{abstract}

PACS numbers: 04.20.Dw, 04.20.Cv, 04.25.Nx, 04.40.Nr, 04.70.Bw

\section{INTRODUCTION}

General relativity is the most successful theory of gravity and it has brought us deep a understanding of spacetime. Nevertheless, when we evolve the Einstein equations with a well-posed initial condition, singularities, at which general relativity and all established theories lose their predictability, are known to form. However, in most cases singularities are hidden by event horizons as in the case of black holes and cannot be seen by a distant observer. It requires extreme fine-tuning of the initial state or unphysical equation of state to produce naked singularities. This statement is known as the cosmic censorship conjecture proposed by Penrose [1]. Despite the tremendous efforts to prove whether the cosmic censorship conjecture is a generic property of classical general relativity, it still remains an open question [2].

According to the uniqueness theorem [3], all stationary asymptotically flat black holes in Einstein-Maxwell system are described by Kerr-Newman solutions, which are specified uniquely by the mass $M$, the charge $Q$ and the angular momentum $J$ satisfying

$$
M^{2} \geq Q^{2}+(J / M)^{2} .
$$

When the equality is saturated, the black holes are called extremal, and a naked singularity appears when $M^{2}<Q^{2}+(J / M)^{2}$. Naively, it seems possible to form a naked singularity by throwing matter into a black hole to increase its charge and angular momentum. If it were really possible, we would say that the black hole is overcharged or overspinned via matter absorption, and it would give a counterexample to the cosmic censorship conjecture.

A number of previous works related to overcharging and overspinning a black hole support the cosmic censorship conjecture. A pioneering work by Wald proved that neither overcharging nor overspinning is possible when a test (charged) particle plunges into an extremal black hole, and hence a naked singularity cannot be produced [4]. If the particle is carrying charge or angular momentum sufficient to overcharge (or overspin) the black hole, it is not captured by the black hole because of the electromagnetic or centrifugal repulsion force. Motivated by Wald's analysis, there have been many analyses attempting to supersaturate an extremal black hole by capturing a particle and a wave packet of a classical field. [5]. All these analyses indicate that such processes at most sustain the extremal condition if the particle is allowed to fall into the black hole. ${ }^{1}$

Recently, however, an alternative viewpoint was raised, suggesting the possibilities of overcharging a Reissner-Nordström (RN) black hole [7], of overspinning a Kerr black hole [8], and of overspinning or overcharging a Kerr-Newmann black hole [9]. The point is to consider the process of particle absorption with the initial black hole being prepared slightly below the extremal

\footnotetext{
*Electronic address: isoyama' at' yukawa.kyoto-u.ac.jp

${ }^{\dagger}$ Electronic address: sago at' yukawa.kyoto-u.ac.jp

‡Electronic address: tanaka' at' yukawa.kyoto-u.ac.jp

1 There is an exception for this statement. De Felice and Yu have pointed out that even an extreme RN black hole can turn into a naked singularity if an electrically neutral spinning particle is sent to an extreme RN black hole with appropriate initial conditions [6].
} 
limit. At a first glance, these works are indicating that the violation of cosmic censorship conjecture is possible. However, as already mentioned in Refs. [7] and [8], the back reaction effects due to the presence of a particle are not taken into account in these analyses. In fact, it was emphasized in Ref. [10] that the finite size effect of a charged particle and the contribution of the interaction energy between the black hole and the particle can be important enough to protect the cosmic censorship conjecture. In Ref. [11], the loss of energy due to gravitational radiation in the spin-up process suggested in Ref. [8] was evaluated. Although the overspinning of a Kerr black hole can be avoided for some parameter choices by taking into account the effect of this radiative loss alone, the possibility of overspinning still remains for the other parameter choices.

Our main purpose of this paper is to clarify the role of back reaction effects in the gedanken experiment discussed in Ref. [7]. Namely, we discuss whether a RN black hole can be overcharged or not by the capturing of a charged particle, taking into account all possible back reaction effects. The basic idea is to concentrate on the case in which the particle is in the orbit at the border between plunge and recoil orbits, which we call the marginal orbit. When the particle takes the marginal orbit, it will experience an unstable equilibrium configuration at the separatrix. Thanks to this property of the marginal orbit, one can use an exact solution of the Einstein-Maxwell equations to read the back reaction effects with sufficient accuracy for this equilibrium configuration. We will show that the total energy of the system is always greater than the total charge. Furthermore, using the black hole perturbation technique, one can evaluate the radiative loss of energy as the particle falls into the black hole from this unstable equilibrium configuration. We will find that the energy loss through this process is always negligible small. As a result, we will conclude that a particle in the marginal orbit cannot overcharge the RN black hole. Naively, the orbits that can plunge into the black hole will have higher energy than the marginal orbit. Hence, our result will indicate that the cosmic censorship conjecture is protected even if we consider the process suggested in Ref. [7].

The outline of this paper is as follows. In Sec. [I] we briefly review the overcharging process suggested in Ref. [7]. After explaining the marginal orbit in Sec. IIII we review the basic properties of an exact solution that corresponds to an unstable stationary equilibrium configuration composed of two charged sources, and we will prove that the total energy is always greater than the total charge for this configuration. In Sec.IV we will show that the radiative energy loss is always suppressed as long as a particle falls from an equilibrium position to the black hole. We summarize the results in Sec. $\mathbf{V}$ adding discussion about the implication of our results.

In this paper, we use the units in which $G=c=1$, but we explicitly write $\kappa^{2}=8 \pi G$ in Sec.IV and Appendix D, respecting the original notations in Ref. [12]. The sign convention of the metric as $(-,+,+,+)$. We adopt the definition of Riemann tensor and Ricci tensor given by $R_{\sigma \mu \nu}^{\rho}:=\partial_{\mu} \Gamma_{\nu \sigma}^{\rho}-\partial_{\nu} \Gamma_{\mu \sigma}^{\rho}+\Gamma_{\mu \lambda}^{\rho} \Gamma_{\nu \sigma}^{\lambda}-\Gamma_{\nu \lambda}^{\rho} \Gamma_{\mu \sigma}^{\lambda}$ and $R_{\mu \nu}:=R_{\mu \alpha \nu}^{\alpha}$. Fourier components of $\Psi(t)$ are defined by $(2 \pi)^{-1 / 2} \int_{-\infty}^{+\infty} e^{i \omega t} \Psi(t) d t$.

\section{OVERCHARGING A REISSNER-NORDSTRÖM BLACK HOLE WITHOUT BACK REACTION EFFECTS}

We briefly review the basic idea of overcharging a RN black hole by the infall of a charged particle, giving the precise meaning of "overcharging", based on the discussion in Ref. [7]. In this section, we first focus on the test particle case in which we neglect the back reaction effects. In this approximation we have a broad range of configurations whose final state exceeds the extremal bound. Later, we discuss how the results are modified by taking into account the back reaction effects.

\section{A. Test particle case}

We consider a point particle with mass $\mu$ and charge $q$ radially falling toward a nearly extremal RN black hole with mass $M$ and charge $Q$. By assumption, these parameters satisfy $\mu<q \ll Q<M$. The background metric and the vector potential of a $\mathrm{RN}$ black hole are given by

$$
\begin{aligned}
d s^{2} & =-f(r) d t^{2}+\frac{1}{f(r)} d r^{2}+r^{2}\left(d \theta^{2}+\sin ^{2} \theta \mathrm{d} \phi^{2}\right), \\
A_{t} & =-\frac{Q}{r}, \quad A_{r}=A_{\theta}=A_{\phi}=0,
\end{aligned}
$$

where

$$
f(r):=1-\frac{2 M}{r}+\frac{Q^{2}}{r^{2}} .
$$

Due to the spherical symmetry of a RN black hole, we can choose the coordinate system such that the trajectory of a particle is along the axis without loss of generality. Then, the trajectory is represented by $z^{\alpha}(s)=(T(s), R(s), 0,0)$ with the proper 
time $s$ along the world line of the particle. The equations of motion of the particle are given by

$$
\begin{aligned}
\left(\frac{d R}{d s}\right)^{2} & =\frac{1}{\mu^{2}}\left(E-\frac{q Q}{R}\right)^{2}-f(R)=: V^{(\mathrm{o})}(R), \\
\left(\frac{d T}{d s}\right) & =\frac{1}{\mu f(R)}\left(E-\frac{q Q}{R}\right) .
\end{aligned}
$$

The energy of the particle $E$ is defined by $E:=-\left(\partial_{t}\right)^{a}\left(\mu u_{a}+q A_{a}\right)$ where $\left(\partial_{t}\right)$ is the Killing field associated with the time coordinate $t$ and $u^{a}:=d z^{a} / d s$ is the four velocity of the particle. Using the $t$-component of the equations of motion (5), it is shown that $E$ is constant along the orbit.

Radial orbits toward the black hole can be classified into three classes: plunge, bounce and marginal orbits. In the case of plunge orbits, a particle falls into the black hole horizon without changing the direction of motion. In the case of bounce orbits, there exists a turning point outside the horizon, at which $(d R / d s)^{2}=0$. The particle is reflected back at this point due to the electric repulsion force. The marginal orbit is on a separatrix between plunge and bounce orbits. In this case, the particle gradually approaches an unstable equilibrium position at $r=r_{0}$. The position of $r_{0}$ and the energy of the marginal orbit are simultaneously determined by the conditions $d R / d s=d^{2} R / d s^{2}=0$.

In the above setup, if the following two conditions are satisfied, we would say that a RN black hole is possibly overcharged. The first condition is that the particle is in a plunge orbit. For plunge orbits there is no turning point where the radial velocity becomes zero. Therefore the condition is

$$
\left(\frac{d R}{d s}\right)^{2}=V^{(\mathrm{o})}(R)>0, \quad \text { for } \quad R \geq r_{+}, \quad \text { (absorption condition) }
$$

where $r_{+}:=M+\sqrt{M^{2}-Q^{2}}$ is the radial coordinate of the event horizon. The second condition is on the total energy of the final state. After the absorption of the particle, the system will approach another RN geometry with mass $M_{\text {final }}=M+E$ and charge $Q_{\text {final }}=Q+q$. The condition that the final RN geometry exceeds the extremal bound is given by

$$
M+E<Q+q . \quad \text { (overcharging condition) }
$$

In Ref. [7], it was demonstrated that radial orbits in a rather wide range of parameter space satisfy the conditions (6) and (7). In fact, both the absorption and overcharging conditions are satisfied for

$$
1<b<a, \quad c<\sqrt{a^{2}-b^{2}},
$$

under the parametrization

$$
M:=1+2 \epsilon^{2}, \quad Q:=1, \quad E:=a \epsilon-2 b \epsilon^{2}, \quad q:=a \epsilon, \quad \mu:=c \epsilon,
$$

where $0<\epsilon \ll 1$ and the coefficients $a, b$ and $c$ are assumed to be $O(1)$ real numbers. Here the overcharging is discussed at the level of $O\left(\epsilon^{2}\right)$, while the mass and the charge of a point particle are $O(\epsilon)$. Although the back reaction effects are completely neglected, the analysis in Ref. [7] is in sharp contrast with previous works [5]. The previous analyses are restricted to $O(\epsilon)$ and only the extremal limit is considered for the initial black hole.

\section{B. Back reaction effects on the overcharging process}

As was already emphasized in Ref. [7], the analysis in the previous section is not sufficient to conclude that a nearly extremal $\mathrm{RN}$ black hole can be overcharged through the absorption of a charged particle. We have to compute the total amount of energy in the final state of the whole system to the accuracy of $O\left(\epsilon^{2}\right)$, taking into account the effects of back reaction [10, 13]. If the evaluation to this accuracy is accomplished, one can judge whether the overcharging condition (7) is satisfied or not. At the same time, we have to examine how the absorption condition (6) is modified once the back reaction effects are taken into account.

To achieve this goal, we need to calculate both the particle motion with the self-force and the self-energy of the system to $O\left(\epsilon^{2}\right)$. However, these quantities have never been computed to such a high order even in the case of Schwarzschild background. Here, we propose to bypass these difficult tasks by focusing on the marginal orbits that pass through an unstable equilibrium state. The basic strategy that we use here is to relate the unstable equilibrium state to an exact solution known as the double Reissner-Nordström static solution [14] (See also Ref. [15]) ${ }^{2}$. Since the latter is an exact solution, it includes all the backreaction effects. Therefore, we can read the total energy of the system in the unstable equilibrium state from this solution.

2 Within the linear perturbation, the global perturbative solution describing the equilibrium configuration of the RN black hole and a charged particle was constructed in Ref. [16]. 
Then, we compute the energy emitted to infinity through the infall of the particle from the equilibrium state to the black hole horizon to the accuracy of $O\left(\epsilon^{2}\right)$, which can be readily achieved using the standard black hole perturbation method. To compute the energy flux emitted to infinity, back reaction effects on the trajectory of a particle can be neglected since they give only higher order corrections to the energy flux. As a result, we obtain a sufficiently accurate estimate for the total energy of the final state for the marginally plunging orbits. In the two successive sections, we prove that the overcharging is impossible for any of such marginal orbits.

To extend our argument to more general cases, we assume that the other plunge orbits result in final states that have higher energies than the final state in the case of the marginal trajectory, whose energy is deduced under the conditions that the mass and charge of the initial black hole and the charge of the plunging particle are fixed. Once we accept this rather reasonable assumption, the avoidance of overcharging in the marginal cases is extended to general plunge orbits. Although, strictly speaking, we cannot verify this last statement, we can at least claim that there is no evidence that supports the possibility of violating the weak cosmic censorship conjecture in the present context.

\section{TOTAL ENERGY OF THE SYSTEM WITH A CHARGED BLACK HOLE AND A CHARGED PARTICLE IN EQUILIBRIUM}

As a first step to examine the overcharging condition (7) for the marginal orbits, we here extract the total energy of the system composed of a charged black hole and a charged particle in the equilibrium state, $E_{\text {eq }}$, using the double Reissner-Nordström (DRN) solution of the Einstein-Maxwell equations [14, 15]. On the other hand, the total charge is simply given by the sum of the charges assingned to respective objects as $Q_{\text {total }}=q+Q$ since the electric charge is not carried by a field. In this section, we will show that the energy at the equilibrium state is always larger than the total charge, $E_{\text {eq }}-(q+Q)=O\left(\epsilon^{2}\right)>0$.

\section{A. The double Reissner-Nordström solution}

We use the form of the DRN solution presented in Ref. [14] in the same notation, except for the signature of the metric, $(-,+,+,+)$. The DRN solution is characterized by five parameters: masses of the RN sources $m_{1}, m_{2}$, their charges $e_{1}, e_{2}$, and the separation between them $\ell$. It should be emphasized that the mass parameters $m_{1}$ and $m_{2}$ do not refer to the rest masses of the RN sources [14]. As we explain later, there is one relation among these five parameters, and hence four of them are independent. With these parameters, the metric and the vector potential are written in the cylindrical Weyl coordinates as

$$
\begin{gathered}
d s^{2}=-H(\rho, z) d t^{2}+F(\rho, z)\left(d \rho^{2}+d z^{2}\right)+\frac{\rho^{2}}{H(\rho, z)} d \phi^{2}, \\
A_{t}=-\Phi(\rho, z), \quad A_{\rho}=A_{z}=A_{\phi}=0 .
\end{gathered}
$$

To express the functions $H, F$ and $\Phi$ in a simple form, we introduce two sets of bipolar coordinates $\left(r_{1}, \theta_{1}\right)$ and $\left(r_{2}, \theta_{2}\right)$ defined by

$$
\left\{\begin{array} { l } 
{ \rho = \sqrt { ( r _ { 1 } - m _ { 1 } ) ^ { 2 } - \sigma _ { 1 } ^ { 2 } } \operatorname { s i n } \theta _ { 1 } , } \\
{ z = z _ { 1 } + ( r _ { 1 } - m _ { 1 } ) \operatorname { c o s } \theta _ { 1 } , }
\end{array} \quad \left\{\begin{array}{l}
\rho=\sqrt{\left(r_{2}-m_{2}\right)^{2}-\sigma_{2}^{2}} \sin \theta_{2}, \\
z=z_{2}+\left(r_{2}-m_{2}\right) \cos \theta_{2}
\end{array}\right.\right.
$$

where $z_{1}$ and $z_{2}$ are set to satisfy $\ell=z_{2}-z_{1}>0$, and $\sigma_{1}^{2}$ and $\sigma_{2}^{2}$ are defined by

$$
\sigma_{1}^{2}=m_{1}^{2}-e_{1}^{2}+2 e_{1} \gamma, \quad \sigma_{2}^{2}=m_{2}^{2}-e_{2}^{2}-2 e_{2} \gamma .
$$

Using these coordinates, the functions $H, F$ and $\Phi$ are written as

$$
\begin{aligned}
& H(\rho, z)=\left[\left(r_{1}-m_{1}\right)^{2}-\sigma_{1}^{2}+\gamma^{2} \sin ^{2} \theta_{2}\right]\left[\left(r_{2}-m_{2}\right)^{2}-\sigma_{2}^{2}+\gamma^{2} \sin ^{2} \theta_{1}\right] \mathcal{D}^{-2}, \\
& F(\rho, z)=\left[\left(r_{1}-m_{1}\right)^{2}-\sigma_{1}^{2} \cos ^{2} \theta_{1}\right]^{-1}\left[\left(r_{2}-m_{2}\right)^{2}-\sigma_{2}^{2} \cos ^{2} \theta_{2}\right]^{-1} \mathcal{D}^{2}, \\
& \Phi(\rho, z)=\left[\left(e_{1}-\gamma\right)\left(r_{2}-m_{2}\right)+\left(e_{2}+\gamma\right)\left(r_{1}-m_{1}\right)+\gamma\left(m_{1} \cos \theta_{1}+m_{2} \cos \theta_{2}\right)\right] \mathcal{D}^{-1},
\end{aligned}
$$

where

$$
\mathcal{D}=r_{1} r_{2}-\left(e_{1}-\gamma-\gamma \cos \theta_{2}\right)\left(e_{2}+\gamma-\gamma \cos \theta_{1}\right)
$$

and the parameter $\gamma$ is defined by

$$
\gamma=\left(m_{2} e_{1}-m_{1} e_{2}\right)\left(\ell+m_{1}+m_{2}\right)^{-1}
$$


The parameter $\sigma_{1}^{2}$ is chosen to be negative while $\sigma_{2}^{2}$ positive. The object labeled with 1 is a naked singularity that has a critical spheroid at $\left\{r_{1}=m_{1}, 0 \leq \rho \leq\left|\sigma_{1}\right|, z=z_{1}\right\}$, while the object labeled with 2 is a black hole surrounded by an event horizon at $\left\{\rho=0, z_{2}-\sigma_{2} \leq z \leq z_{2}+\sigma_{2}\right\}$. The position of the naked singularity is in the region $r_{1}<m_{1}$, which is not covered by the original coordinates $(\rho, z)$. To guarantee the existence of an equilibrium state without any conical singularities between the two objects, a balance condition,

$$
m_{1} m_{2}=\left(e_{1}-\gamma\right)\left(e_{2}+\gamma\right),
$$

must be satisfied. This gives one relation among five parameters $\left(m_{1}, m_{2}, e_{1}, e_{2}, \ell\right)$, which can be satisfied only when $\sigma_{1}^{2} \sigma_{2}^{2} \leq 0$. Namely, the DRN solution should consist either of a black hole and a naked singularity, or of two extremal black holes. The formar case requires that $\sigma_{1}^{2}$ and $\sigma_{2}^{2}$ should have opposite signs, as we have chosen above. The latter is a special case of the Majumdar-Papapetrou solution [17].

The total mass and charge of the DRN solution can be read from the asymptotic forms of the metric functions (14) and (15), and the electric potential (16). At the space-like infinity, defined by the limit $\rho \rightarrow \infty$ or $z \rightarrow \infty$, the two bipolar coordinates $\left(r_{1}, \theta_{1}\right)$ and $\left(r_{2}, \theta_{2}\right)$ coincide with the spherical coordinates $(r, \theta)$ defined by $\rho=r \sin \theta$ and $z=r \cos \theta$. Thus, the metric functions and the electric potential are expanded as:

$$
\begin{aligned}
H & =1-\frac{m_{1}+m_{2}}{r}+O\left(\frac{1}{r^{2}}\right), \\
F^{-1} & =1-\frac{m_{1}+m_{2}}{r}+O\left(\frac{1}{r^{2}}\right), \\
\Phi & =\frac{e_{1}+e_{2}}{r}+O\left(\frac{1}{r^{2}}\right),
\end{aligned}
$$

in this region. For asymptotically flat spacetime, the coefficients of the $O(1 / r)$ terms of the metric function and the electric potential correspond to the total energy and charge of the system, respectively. Thus we find that the total energy is $m_{1}+m_{2}$ and the total charge is $e_{1}+e_{2}$.

\section{B. Mass dominance of the DRN solution}

In this subsection, we study the DRN solution consisting of a black hole with charge $e_{2}$ and mass $m_{2}$ satisfying $\sigma_{2}^{2}>0$, and a naked singularity with charge $e_{1}$ and mass $m_{1}$ satisfying $\sigma_{1}^{2}<0$. We assume

$$
m_{2}>m_{1}>0, \quad e_{2}>e_{1}>0,
$$

which means that the mass and the charge of the black hole are larger than those of the naked singularity. Under these conditions, we show that the total mass is larger than the total charge, i.e. $m_{1}+m_{2}>e_{1}+e_{2}$.

To this end, we re-express the balance equation (19) as

$$
I(\gamma):=\gamma^{2}+\left(e_{2}-e_{1}\right) \gamma+m_{1} m_{2}-e_{1} e_{2}=0 .
$$

In addition, from $\sigma_{1}^{2}<0, \sigma_{2}^{2}>0$, and using Eq. [13), we obtain the conditions for the parameter $\gamma$ as

$$
\gamma<\frac{e_{1}^{2}-m_{1}^{2}}{2 e_{1}}, \quad \gamma<\frac{m_{2}^{2}-e_{2}^{2}}{2 e_{2}},
$$

and, from the positivity of the separation $\ell>0$ and using Eq. [18), we find

$$
\begin{cases}\frac{m_{2} e_{1}-m_{1} e_{2}}{m_{1}+m_{2}}<\gamma<0, & \text { for } m_{2} e_{1}-m_{1} e_{2}<0, \\ 0<\gamma<\frac{m_{2} e_{1}-m_{1} e_{2}}{m_{1}+m_{2}}, & \text { for } m_{2} e_{1}-m_{1} e_{2}>0 .\end{cases}
$$

In this context, the balance condition (22) is interpreted as the condition that the quadratic equation of $\gamma$ has a solution in the range specified by the conditions, (23) and (24).

Now we examine if a solution exists for three cases: (i) $\gamma<0$ and $m_{1} m_{2}>e_{1} e_{2}$, (ii) $\gamma<0$ and $m_{1} m_{2}<e_{1} e_{2}$, and (iii) $\gamma>0$ and $m_{1} m_{2}<e_{1} e_{2}$. For $\gamma>0$, the case with $m_{1} m_{2}>e_{1} e_{2}$ is immediately excluded by the condition (22).

Case (i): In this case, the conditions (23) and (24) imply

$$
-\gamma>\frac{m_{1}^{2}-e_{1}^{2}}{2 e_{1}}, \quad-\gamma>\frac{e_{2}^{2}-m_{2}^{2}}{2 e_{2}}, \quad-\gamma<\frac{m_{1} e_{2}-m_{2} e_{1}}{m_{1}+m_{2}} .
$$


The first two inequalities in 25] lead to

$$
\begin{aligned}
-\gamma & >\frac{1}{m_{1}+m_{2}}\left[\frac{m_{2}\left(m_{1}^{2}-e_{1}^{2}\right)}{2 e_{1}}+\frac{m_{1}\left(e_{2}^{2}-m_{2}^{2}\right)}{2 e_{2}}\right] \\
& =\frac{m_{1} e_{2}-m_{2} e_{1}}{2\left(m_{1}+m_{2}\right)}\left[1+\frac{m_{1} m_{2}}{e_{1} e_{2}}\right] \\
& >\frac{m_{1} e_{2}-m_{2} e_{1}}{m_{1}+m_{2}}
\end{aligned}
$$

where we used $m_{1} m_{2}>e_{1} e_{2}$ in the last line. However, the inequality (26) is in contradiction with the last condition in (25). Thus, case (i) is excluded.

Case (ii): In this case, the balance condition (22) implies

$$
\gamma^{2}+\left(e_{2}-e_{1}\right) \gamma=e_{1} e_{2}-m_{1} m_{2}>0,
$$

which leads to the inequality

$$
\gamma+\left(e_{2}-e_{1}\right)<0
$$

Combining this inequality with Eqs. (24), we find

$$
\frac{m_{1} e_{2}-m_{2} e_{1}}{m_{1}+m_{2}}>-\gamma>e_{2}-e_{1}
$$

and hence we have

$$
m_{1} e_{1}>m_{2} e_{2}
$$

However, the last inequality $m_{1} e_{1}>m_{2} e_{2}$ is in contradiction with the assumption $m_{2}>m_{1}>0$ and $e_{2}>e_{1}>0$. Thus, case (ii) is also excluded.

Case (iii): Recalling that $I(0)=m_{1} m_{2}-e_{1} e_{2}<0$, the necessary condition that $I(\gamma)=0$ has a positive root satisfying the condition (24) is

$$
I\left(\frac{m_{2} e_{1}-m_{1} e_{2}}{m_{1}+m_{2}}\right)=\frac{m_{1} m_{2}}{\left(m_{1}+m_{2}\right)^{2}}\left[\left(m_{1}+m_{2}\right)^{2}-\left(e_{1}+e_{2}\right)^{2}\right]>0 .
$$

This inequality leads to

$$
m_{1}+m_{2}>e_{1}+e_{2}
$$

Since both case (i) and case (ii) are not allowed, the only possibility is case (iii), and we also find that $\gamma$ cannot be negative simultaneously. In case (iii) the inequality (32) was proven. Hence, to conclude, under the assumptions $m_{2}>m_{1}>0$ and $e_{2}>e_{1}>0$, we have shown that

$$
E_{\mathrm{eq}}>q+Q
$$

which means that the total energy never goes below the extremal bound in the equilibrium configuration.

\section{Remarks on the use of the DRN solution}

In the preceding subsection, we used the DRN solution as such that describes an equilibrium state of a charged particle in a black hole spacetime. However, strictly speaking, we also have to prove that the charged singularity in the DRN solution is a valid approximation for a charged particle. An arbitrary solution that allows a singularity may in general possess higher multipole moments other than the monopole. In that case the charged particle will have an extra self-energy due to higher multipole distortion. Thus, one may suspect that there might be another equilibrium configuration that has lower total energy with the same total charge. Hence, it would be necessary to explicitly demonstrate that the charged particle in the DRN solution does not possess higher order multipole moments that are large enough to contribute to the total energy of $O\left(\epsilon^{2}\right)$. A detailed discussion is given in Appendix $\mathrm{A}$ and the outline is as follows. We first show that the behavior near the singularity in the DRN solution can be described by a perturbed RN configuration. Perturbative expansion does not break down even near the singularity in the DRN solution. From this expression we can read the maximum amplitude of the multipole moments that the particle possesses. The largest component is the dipole and its magnitude is $O\left(\epsilon^{3}\right)$, and we find that the contribution of such a dipole moment to the total energy is at most $O\left(\epsilon^{3}\right)$. 


\section{ENERGY FLUX RADIATED FROM A CHARGED PARTICLE}

In the preceding section, with the help of the DRN solution, we showed that the total energy of the equilibrium state of a charged particle in a RN black hole cannot be less or equal to its total charge. This suggests that the system in equilibrium does not exceed the extremal bound, but it is not sufficient yet to exclude the possibility that a RN black hole is overcharged by absorbing a charged particle. The effects of the electromagnetic and gravitational radiation can reduce the total energy as the particle falls from the equilibrium position to the event horizon of the black hole. Then the total energy in the final state will be given by $E_{\text {eq }}-E_{\infty}$ with $E_{\infty}$ being the energy radiated away to infinity. In this section, we estimate $E_{\infty}$ using linear perturbation theory. We examine if the signature of $E_{\text {eq }}-q-Q-E_{\infty}$ can be negative, to see whether the overcharging in the final configuration is possible or not.

\section{A. Correspondence between two different pictures}

Before evaluating the radiation energy emitted to infinity, we would like to briefly mention the correspondence between the DRN solution and the equilibrium configuration of a test particle in a RN black hole. Here we refer to the latter as the black hole perturbation (BHP) picture. Our current interest is in the case in which the charge of the test particle is $O(\epsilon)$, while the difference between the total energy and the total charge is $O\left(\epsilon^{2}\right)$ :

$$
e_{1}:=a \epsilon, \quad e_{2}:=1, \quad m_{1}:=O(\epsilon), \quad m_{2}:=1+\varpi(1+O(\epsilon)), \quad\left(m_{1}+m_{2}\right)-\left(e_{1}+e_{2}\right)=O\left(\epsilon^{2}\right) .
$$

As a starting point we do not exclude the possibility that $\varpi$ is as large as $O(\epsilon)$. There might be more subtle cases if we consider the possibility that the difference $\left(m_{1}+m_{2}\right)-\left(e_{1}+e_{2}\right)$ is $O\left(\epsilon^{3}\right)$ or higher, but such cases are beyond the scope of the present paper.

Then, as shown in Appendix $B$, the parameters used to describe the DRN solution are expanded in terms of $\epsilon$ as

$$
e_{1}=a \epsilon, \quad e_{2}=1, \quad \gamma=g \epsilon^{2}, \quad \ell \gamma=\tilde{g} \epsilon^{3},
$$

where $a$ and $\tilde{g}$ are expected to be $O(1)$, while $g$ can be either $O(1)$ or even smaller. If $g$ is of higher order in $\epsilon, \ell$ should be large so as to maintain $\ell \gamma=O\left(\epsilon^{3}\right)$. The meaning of $a$ is obviously identical to that in the BHP picture. Substituting Eqs. (35) into Eqs. (13), (18) and (19), we obtain approximations for the mass parameters $\left(m_{1}, m_{2}\right)$ and $\left(\sigma_{1}, \sigma_{2}\right)$ as

$$
m_{1}=a \epsilon-g \epsilon^{2}-\frac{\tilde{g}}{2} \epsilon^{3}, \quad m_{2}=1+\left(g+\frac{\tilde{g}}{2 a}\right) \epsilon^{2}, \quad \sigma_{1}^{2}=\left(g^{2}-a \tilde{g}\right) \epsilon^{4}, \quad \sigma_{2}^{2}=\frac{\tilde{g}}{a} \epsilon^{2},
$$

where the terms of higher order in $\epsilon$ are neglected. The relations (36) enable us to evaluate the difference between the total energy and the total charge as

$$
m_{1}+m_{2}-\left(e_{1}+e_{2}\right)=\frac{\tilde{g}}{2 a} \epsilon^{2}+O\left(\epsilon^{3}\right),
$$

which is positive as long as $\gamma$ and $\ell$ are positive. This is, of course, consistent with the more general argument given in the preceding section.

The relation between the parameters $\tilde{g}$ and $g$ in the DRN solution and the parameters in the BHP picture is examined in Appendix $\mathrm{C}$, and is given by

$$
\tilde{g}=4 a+O(\epsilon), \quad g=2 \sqrt{a^{2}-c^{2}}+O(\epsilon)=2 b+O(\epsilon) .
$$

(Here $b$ is simply determined by the equilibrium condition C1.) From these expressions we can confirm that there are counterparts of $g$ and $\tilde{g}$ for any values of $a$ and $c$. Using the above relation, we finally obtain

$$
m_{1}+m_{2}-\left(e_{1}+e_{2}\right)=2 \epsilon^{2}+O\left(\epsilon^{3}\right) .
$$

In the analysis neglecting all back reaction effects, as in Ref. [7], our interest was restricted to the parameter region with $b>1$ that allows overcharging in the final state. Under this restriction, $g$ must be definitely $O(1)$. As a result, $\ell$ must be $O(\epsilon)$. As shown in Appendix. C the corresponding proper separation is $\mathrm{O}(1)$. However, now we know that, once all the back reaction effects are taken into account, the difference between the total energy and the total charge at the equilibrium configuration is always positive and of $O\left(\epsilon^{2}\right)$ in our setup of the problem. Hence, if the effect of energy loss due to radiation is less than $O\left(\epsilon^{2}\right)$, there is no possibility of overcharging. To the contrary, if the energy loss is as large as $O\left(\epsilon^{2}\right)$, all parameter region in principle may give an example of overcharging. Hence, in the succeeding discussion we will not restrict ourselves to the case with $b=O(1)$. From the above correspondence, we understand that, when $b$ is of higher order, so is $g$, which corresponds to the case that $\ell$ is greater than $O(\epsilon)$. In this case, the difference in the rest mass and the charge of the point particle $a-c$ is also higher order and the proper separation between the horizon and the particle is as large as $O(|\log \epsilon|)$. 


\section{B. Gauge invariant perturbation of the $\mathrm{RN}$ black hole excited by a charged particle}

To estimate the energy emitted to infinity by the electromagnetic and gravitational radiation, we use linear perturbation theory, which has been developed for a RN spacetime in Refs. [12, 18]. Here we adopt the formulation developed by Kodama and Ishibashi [12], in which the equations are reduced to a set of decoupled second-order differential equations.

Owing to the background spherical symmetry, one can decompose the perturbations into scalar- and vector-types in general. It is easy to check that the energy-momentum tensor of a charged particle in a radial orbit is purely of scalar-type, and therefore only the scalar-type perturbations are excited. In addition to that, by choosing the coordinates so that the trajectory is along the axis we can restrict our analysis to the axisymmetric mode $\mathfrak{m}=0$, where $\mathfrak{m}$ is the azimuthal index of spherical harmonics $Y_{\mathfrak{l m}}(\theta, \phi)$. As shown in Ref. [12], the equations of the scalar-type perturbation are reduced to two decoupled equations for two gauge invariant variables, $\Phi_{ \pm}$. They are given in terms of Fourier-harmonics expansion as

$$
\frac{d^{2} \Phi_{ \pm}\left(r^{*}\right)}{d r^{* 2}}+\left(\omega^{2}-V_{ \pm}(r)\right) \Phi_{ \pm}\left(r^{*}\right)=S_{ \pm}\left(r^{*}, \omega\right)
$$

where the tortoise coordinate $r_{*}$ is defined by $d r_{*} / d r=1 / f(r)$, when $f(r)$ is as defined in Eq. (3), and $\omega$ is the frequency. For simplicity, we abbreviate the labels $\mathfrak{l}, \mathfrak{m}, \omega$ to be attached to the master variables, effective potentials and source terms. The effective potentials, $V_{ \pm}(r)$, are defined by

$$
V_{ \pm}(r):=\frac{f(r) U_{ \pm}(r)}{64 r^{2} H_{ \pm}^{2}(r)}
$$

with auxiliary functions

$$
H_{+}:=1-3 \delta x, \quad H_{-}:=m+3 \rho x, \quad \delta:=\frac{1}{2 m}\left(\frac{\nu}{M}-1\right), \quad \nu^{2}:=M^{2}+\frac{4 m Q^{2}}{9}, \quad \rho:=1+m \delta,
$$

where $x:=2 M / r$ and $z:=Q^{2} / r^{2}$. Here we introduced $m:=\mathfrak{l}(\mathfrak{l}+1)-2$ where $\mathfrak{l}(\mathfrak{l}+1)$ is the eigenvalue of the Laplace operator on $S^{2}$. (It should be stressed that $m$ is different from the azimuthal index $\mathfrak{m}$ ). The functions $U_{ \pm}(r)$ in Eq. (41) are given by

$$
\begin{aligned}
& U_{+}(r):=-2592 \delta^{3} \rho x^{4}+576(3 m \delta+4) \delta^{2} x^{3}-192 \delta x(3 x+m)+64(m+2) \\
& U_{-}(r):=-2592 \delta \rho^{3} x^{4}-576(3 m \delta-1) \rho^{2} x^{3}+192 m \rho x(3 x+m)+64 m^{2}(m+2) .
\end{aligned}
$$

The tensor composed of the scalar-type harmonics vanishes for $\mathfrak{l}=1$, or equivalently for $m=0$. This mode is called an exceptional mode in Ref. [12], which requires special treatment. In this case, although the master equation (40) is still valid, the dynamical variable is $\Phi_{+}$only. For the exceptional mode, $\delta$ takes the value $Q^{2} / 9 M^{2}$, which is obtained by taking the limit $m \rightarrow 0$.

The source terms $S_{ \pm}\left(r^{*}, \omega\right)$ are constructed from the energy-momentum tensor and the current induced by a charged particle. They are given by

$$
S_{ \pm}\left(r^{*}, \omega\right):=a_{ \pm}(r) S_{\Phi}(r, \omega)+b_{ \pm} S_{\mathcal{A}}(r, \omega)
$$

with the coefficients defined by

$$
\begin{aligned}
& \left\{a_{+}(r), b_{+}\right\}:=\left\{\frac{m Q}{2}+\frac{3(M+\nu) Q}{2 r}, \frac{3(M+\nu) \kappa}{\sqrt{2}}\right\}, \\
& \left\{a_{-}(r), b_{-}\right\}:=\left\{3(M+\nu)-\frac{4 Q^{2}}{r},-4 \sqrt{2} Q \kappa\right\},
\end{aligned}
$$

and

$$
\begin{aligned}
S_{\Phi}(r, \omega):= & \frac{\sqrt{2} f Q \kappa}{r^{3} H}\left[\left\{-\frac{P_{S 1}}{H}+2(m+2)\right\} \frac{\tilde{J}_{t}}{i \omega}\right] \\
& +\frac{f}{r H}\left(\frac{P_{S 3}}{H} \frac{r S_{t}^{r}}{i \omega}+2 r^{2} \frac{1}{i \omega} \frac{\partial S_{t}^{r}}{\partial r}+2 r^{2} S_{r}^{r}\right), \\
S_{\mathcal{A}}(r, \omega):= & -\left(\frac{8 z f^{2}}{r^{2} H}-\omega^{2}\right) \frac{\tilde{J}_{t}}{i \omega}-f \frac{\partial}{\partial r}\left(f \tilde{J}_{r}\right)-\frac{2 \sqrt{2} f Q}{i \omega H \kappa} \frac{S_{t}^{r}}{r} .
\end{aligned}
$$


Here functions $H, P_{S 1}$ and $P_{S 3}$ are defined by

$$
H:=m+3 x-4 z, \quad P_{S 1}:=4 x[2 z-3 x+6+m(m+4)], \quad P_{S 3}:=4(3 x-8 z),
$$

$S_{a}^{b}(t)$ and $\tilde{J}_{a}(t)$ are respectively the Fourier components of the energy momentum tensor and the charge current of a radially falling charged particle that starts with a stationary point $r=r_{0}$ in the infinite past $t \rightarrow-\infty$. Their explicit forms are expressed as

$$
\begin{aligned}
S_{t}^{r} & =-\kappa^{2} \mu f\left(\frac{d T}{d s}\right) \frac{e^{i \omega T}}{r^{2}} Y_{\mathfrak{l} 0} \theta\left(r_{0}-r\right), \quad S_{r}^{r}=\kappa^{2} \mu f^{-1}\left(\frac{d R}{d s}\right) \frac{e^{i \omega T}}{r^{2}} Y_{\mathfrak{l} 0} \theta\left(r_{0}-r\right), \\
\tilde{J}_{t} & =\frac{q}{\mathfrak{l}(\mathfrak{l}+1)} e^{i \omega T} Y_{\mathfrak{l} 0} \theta\left(r_{0}-r\right), \quad \tilde{J}_{r}=-\frac{q}{\mathfrak{l}(\mathfrak{l}+1)}\left(\frac{d T}{d R}\right) e^{i \omega T} Y_{\mathfrak{l} 0} \theta\left(r_{0}-r\right),
\end{aligned}
$$

where $Y_{\mathfrak{I} 0}=\sqrt{(2 \mathfrak{l}+1) / 4 \pi}$. In these expressions $d T / d s, d R / d s, d T / d R:=(d T / d s) /(d R / d s)$ and $T$ are to be understood as functions of $R$, and $R$ is replaced with $r$. The function $T$ is obtained by integrating Eqs. (4) and (5) and eliminating $s$.

The formal solutions $\Phi_{ \pm}$of Eq. (40) applicable to both the generic and exceptional modes are obtained by the usual Green function method. The asymptotic form of the solution at $r \rightarrow+\infty$ is given by

$$
\Phi_{ \pm}\left(r^{*}, \omega\right)=\frac{e^{i \omega r^{*}} \chi_{ \pm}(\omega)}{W\left[\Phi_{ \pm}^{\mathrm{up}}, \Phi_{ \pm}^{\mathrm{in}}\right]}
$$

with

$$
\chi_{ \pm}(\omega):=\int_{r_{+}^{*}}^{r_{0}^{*}} \Phi_{ \pm}^{\mathrm{in}}\left(r^{*}, \omega\right) S_{ \pm}\left(r^{*}, \omega\right) d r^{*}
$$

where the functions $\Phi_{ \pm}^{\mathrm{up} / \mathrm{in}}\left(r^{*}, \omega\right)$ are the homogeneous solutions of Eq. (40) that satisfy the boundary conditions $\Phi_{ \pm}^{\mathrm{up}}\left(r^{*}, \omega\right) \rightarrow$ $e^{i \omega r^{*}}$ at $r^{*} \rightarrow \infty$ and $\Phi_{+}^{\text {in }}\left(r^{*}, \omega\right) \rightarrow e^{-i \omega r^{*}}$ at $r^{*} \rightarrow-\infty$, respectively. We also introduced the Wronskian defined by $W\left[\Phi^{1}, \Phi^{2}\right]:=\left(\partial_{r^{*}} \Phi^{1}\left(r^{*}\right)\right) \Phi^{2}\left(r^{*}\right)-\Phi^{1}\left(r^{*}\right)\left(\partial_{r^{*}} \Phi^{2}\left(r^{*}\right)\right)$.

The energy flux carried by electromagnetic and gravitational waves to infinity is described in terms of the variables $\Phi_{ \pm}$ as given in Eq. (D5) in Appendix D In this expression $\bar{\Phi}_{ \pm}(r, t)$ are the complex conjugations of $\Phi_{ \pm}(r, t)$. Substituting the solution (51) into the time integral of Eq. (D5), with the aid of Parseval's theorem, we obtain the total energy radiated to infinity

$$
E_{\infty}=\int_{0}^{+\infty} d \omega \sum_{\mathfrak{l}} \frac{8 \pi \mathfrak{l}(\mathfrak{l}+1) \omega^{2}}{9 \kappa^{2} \nu(M+\nu)}\left(\frac{\left|X_{+}\right|^{2}}{\left|W_{+}\right|^{2}}+\frac{(\mathfrak{l}-1)(\mathfrak{l}+2)}{16} \frac{\left|X_{-}\right|^{2}}{\left|W_{-}\right|^{2}}\right),
$$

where $W_{ \pm}:=W\left[\Phi_{ \pm}^{\mathrm{up}}, \Phi_{ \pm}^{\mathrm{in}}\right]$. The explicit form of $\mathcal{X}_{ \pm}$is given in Eq. [D9]. We point out that the integrals in the expressions for $x_{ \pm}$given in Eq. (D9), with the last two terms replaced with Eq. (D11) all take the form

$$
\int_{r_{+}}^{r_{0}} I(r) \Phi_{ \pm}^{\mathrm{in}}(r) e^{i \omega T(r)} d r
$$

where $I(r)$ is a certain regular function of $r$, whose typical scale of variation $\Delta r$ is $O\left(M^{-1}\right)$. For large $\omega$, the integrals (53) take the structure of rapidly oscillating function $\Phi_{ \pm}^{\mathrm{in}}(r) e^{i \omega T(r)}$ multiplied by the slowly varying function $I(r)$. In general, such an integral is known to be exponentially suppressed for large $M \omega$. Thus, the $\omega$-integral in Eq. (52) has an effective high frequency cutoff at $O\left(M^{-1}\right)$.

Now we show that this radiated energy is $O\left(\epsilon^{4}\right)$ or higher. It is obvious that $\left|X_{ \pm}(\omega)\right|$ is suppressed by a factor of $O(\epsilon)$ since each term manifestly contains $\mu$ or $q$. Therefore what we have to show is that there is an additional suppression of $O(\epsilon)$ in $\left|x_{ \pm}(\omega)\right|$.

As a preparation, we show that the inverse of the Wronskian is suppressed in the limit $\omega \rightarrow 0$ like $W_{ \pm}^{-1} \propto(M \omega)^{\mathfrak{l}}$. The presence of this suppression is understood as follows. Since the Wronskian is constant independently of $r$, we evaluate it in the limit $r \rightarrow \infty$ as

$$
W_{ \pm}=\lim _{r \rightarrow \infty}\left(i \omega \Phi_{ \pm}^{\mathrm{in}}(r)-\frac{\partial \Phi_{ \pm}^{\mathrm{in}}(r)}{\partial r}\right) e^{i \omega r}
$$

Note that in the limit $r \rightarrow \infty$, the difference between $r^{*}$ and $r$ is suppressed by $1 / r$, so that we can use $r$ instead of $r^{*}$ in Eq. (54). When $r$ is large enough compared with $M$, the homogeneous equation corresponding to Eq. (40) can be approximated as

$$
\frac{d^{2} \Phi_{ \pm}(r)}{d r^{2}}+\left(\omega^{2}-\frac{\mathfrak{l}(\mathfrak{l}+1)}{r^{2}}\right) \Phi_{ \pm}(r)=0 .
$$


The general solution of this equation, which is written in terms of the Bessel function of the first kind $J_{\mathfrak{l}}$ and that of the second kind $Y_{\mathfrak{l}}$, should also describe $\Phi_{ \pm}^{\text {in }}$. Hence, we have

$$
\Phi_{ \pm}^{\text {in }} \approx C_{J} \sqrt{\frac{\pi \omega r}{2}} J_{\mathfrak{l}+1 / 2}(r \omega)+C_{Y} \sqrt{\frac{\pi \omega r}{2}} Y_{\mathfrak{l}+1 / 2}(r \omega)
$$

where $C_{J}$ and $C_{Y}$ are coefficients to be determined by the condition imposed near the horizon. Then, for a small $r$ of $O(M)$ on the verge of the validity of this approximate solution, the two terms in Eq. 56 should be equally important in general. Furthermore, as there is no significant feature in the potential, the amplitude of these terms must be $O(1)$. This determines the order of magnitude of the coefficients as $C_{J}=O\left((M \omega)^{-\mathfrak{l}-1}\right)$ and $C_{Y}=O\left((M \omega)^{\mathfrak{l}}\right)$, since for a small $r \omega$ the above expression asymptotically behaves as

$$
\Phi_{ \pm}^{\text {in }} \rightarrow C_{J} \sqrt{\frac{\pi}{2}}\left(\frac{\omega r}{2}\right)^{\mathfrak{l}+1} \frac{1}{\Gamma(\mathfrak{l}+3 / 2)}-C_{Y} \frac{1}{\sqrt{2 \pi}}\left(\frac{\omega r}{2}\right)^{-\mathfrak{l}} \Gamma(-\mathfrak{l}-1 / 2) .
$$

On the other hand, for a large $r$, the first term dominates to give

$$
\Phi_{ \pm}^{\text {in }} \rightarrow C_{J} \cos \left(\omega r-\frac{\pi}{2}(\mathfrak{l}+1)\right) .
$$

Substituting this expression into Eq. (54), the order of magnitude of the Wronskian is estimated as $W_{ \pm}=O\left(\frac{1}{M}(M \omega)^{-\mathfrak{l}}\right)$. As anticipated, we find that the inverse of the Wronskian scales like $\propto(M \omega)^{\mathfrak{l}}$.

Now, in order to prove the presence of an additional suppression factor in $\left|X_{ \pm}(\omega)\right|$, we focus on the fact that $(d R / d s)^{2}=V^{(\mathrm{o})}$ is always suppressed in the present setup. It will be obvious that $V^{(\mathrm{o})}(r)$ is a quadratic function of $1 / r$ bounded from below. Furthermore, we know that both $V^{(\mathrm{o})}$ and $d V^{(\mathrm{o})} / d r$ vanishes at $r=r_{0}$. Therefore $V^{(\mathrm{o})}$ takes its maximum value at $r=r_{+}$, in the interval of our interest between $r_{+}$and $r_{0}$. Hence, we have

$$
V^{(\mathrm{o})}(r) \leq V^{(\mathrm{o})}\left(r_{+}\right)=\frac{1}{\mu^{2}}\left(E-\frac{q Q}{M+\sqrt{M^{2}-Q^{2}}}\right)^{2}=\frac{4(a-c)^{2}}{c^{2}} \epsilon^{2}+O\left(\epsilon^{3}\right) .
$$

This implies that the velocity of a particle $d R / d s$ given in Eq. (4) is always at most $O(\epsilon)$. When the particle moves very slowly, the amount of emitted radiation is also expected to be small. This intuition can be made explicit in the expression for $X_{ \pm}$as follows. One can see that each expression in Eq. (53) is regular on the boundaries of the integral. Here we replace $e^{i \omega T(r)}$ with an equivalent expression

$$
\frac{f}{i \omega}\left(\frac{d R}{d s}\right)\left(f \frac{d T}{d s}\right)^{-1}\left(\frac{\partial e^{i \omega T(r)}}{\partial r}\right)
$$

and perform integration by parts. The regularity on the boundaries of the integral is not ruined thanks to the presence of a factor $f$ and $d R / d s$ in the above expression 60). (Notice that the combination $f(d T / d s)$ is regular on the horizon.) This manipulation adds at least one $d R / d s$ factor at the expense of decreasing the power of $\omega$ by one. Repeated application of this integration by parts is restricted by the requirement for the convergence of the $\omega$-integral in Eq. 52. However, this is not such a severe constraint, because the inverse Wronskian squared $\left|W_{ \pm}\right|^{-2}$ gives suppression for a small $\omega$ proportional to $\omega^{2 \mathfrak{l}}$ as mentioned above. Therefore this additional suppression owing to the $d R / d s$ factor guarantees that the total energy emitted to infinity is at most $O\left(\epsilon^{4}\right)$.

To summarize, the total energy emitted to infinity by a particle that falls from the unstable stationary point to the horizon, $E_{\infty}$, is always suppressed, and it cannot be as large as $O\left(\epsilon^{2}\right)$ for any parameter choice. Hence, the effect of the energy loss due to radiation does not affect the inequality 37 , which is a relation at the level of $O\left(\epsilon^{2}\right)$. Thus, we conclude that

$$
E_{\mathrm{eq}}-E_{\infty}>q+Q
$$

is always satisfied. Namely, the total mass of the final state can never be reduced below the critical value that corresponds to the extremal bound.

\section{CONCLUSION}

In this work, we have examined the back reaction effects of $O\left(\epsilon^{2}\right)$ when a charged particle whose mass and charge are of $O(\epsilon)$ is absorbed by a nearly extremal RN black hole. To avoid the technical difficulties related to the electromagnetic and 
gravitational self-force, we concentrated on the case of the marginal orbit, which is the separatrix dividing the plunge and recoil orbits. We first showed that, with the aid of an exact solution, the total energy of the system is always greater than the total charge for the equilibrium configuration that the marginal orbit passes through. Then, we demonstrated that the radiative energy loss as the particle is falling into the black hole from the equilibrium position is $O\left(\epsilon^{4}\right)$ or higher. Combining these results, we succeeded in proving that the total energy of the system composed of a charged particle and a black hole is always greater than their total charge for the marginal orbit, once back reaction effects are properly taken into account. In short, the back reaction effects prevent a nearly extremal RN black hole from being overcharged, and hence we conclude the cosmic censorship conjecture is not violated.

As was mentioned in Sec. IIB our discussion relies on the assumption that the marginal orbit passes through the unstable stationary configuration. Although it is difficult to imagine that this is not the case, our modest conclusion at the moment will be that there is no evidence that supports the possibility of overcharging a nearly extremal RN black hole by absorbing a charged particle. If we further accept a rather natural assumption that the final state of all the plunging orbits has a higher energy than the case of the marginal orbit for given any charges of particle and black hole, we can exclude the possibility of overcharging without the restriction to the marginal orbit.

To obtain a definitive answer to the question whether these assumptions are correct or not, it is necessary to solve the orbital evolution directly including the self-force effects. The formal expressions for the gravitational self-force in vacuum spacetime was already derived by Mino, Sasaki and Tanaka, and independently by Quinn and Wald [19], and is known as the MiSaTaQuWa force. In the case of a charged particle in the non-vanishing electro-magnetic background, even the fundamental formulation corresponding to the MiSaTaQuWa force is lacking. Although there is a recent work in this direction [20], it has not yet been developed to the level applicable to the overcharging problem discussed in this paper.

The main lesson of this paper is that it is important to include the non-dissipative part of the self-force when we examine the possibility of overcharging. In the context of the spinning up of a Kerr black hole proposed in Ref. [8], Barausse et al. have shown that, to avoid overspinning, it is not sufficient to take into account only the dissipative part of the self-force, i.e. the energy loss and the angular momentum loss due to gravitational radiation [11]. As was discussed in Ref. [11], also in this case it is essential to take into account the non-dissipative part of the self-force. However, in contrast to the overcharging process discussed in this paper, we cannot expect the existence of the stationary intermediate configuration characterizing the marginal orbit. Therefore, directly analysis of the self-force will be unavoidable.

\section{Acknowledgments}

It is our pleasure to thank Tetsuya Shiromizu for his valuable comments, especially for pointing out the potential existence of subtle issues in using the DRN solution as we discussed in Appendix A We also wish to thank Akihiro Ishibashi, Masashi Kimura Shunichiro Kinoshita, Norihiro Tanahashi and Chul-Moon Yoo for fruitful discussions. We also grateful to Jonathan White for his careful reading of the manuscript, which is very useful to improve the presentation. N. S. and T. T. acknowledge support by the Grant-in-Aid for Scientific Research (No. 21244033). This work was supported by the Grant-in-Aid for the Global COE Program "The Next Generation of Physics, Spun from Universality and Emergence" from the Ministry of Education, Culture, Sports, Science and Technology of Japan.

\section{Appendix A: Geometry near the singularity in the DRN solution}

In Sec. IIIC we implicitly assumed that the singularity in the DRN solution is the counterpart of a charged particle. The aim of this appendix is to confirm this correspondence. The main focus is on the asymptotic behavior near the singularity, so as to show that the deformation from the spherically symmetric singularity is sufficiently small.

To analyze the geometry near the singularity in the DRN solution, the original $(\rho, z)$ coordinates are inappropriate since they do not cover the region where the singularity resides. In order to analytically continue DRN metric (10), we can use the bipolar coordinates $\left(r_{1}, \theta_{1}\right)$ defined by Eqs. (12). However, to see the behavior near the singularity, it is more convenient to change the radial coordinate from $r_{1}$ to $\mathcal{D}$. Furthermore, as we are focusing on the behavior near the singularity, we use the rescaled variable $\Delta$ defined by $\mathcal{D}=\Delta \epsilon^{2}$, instead of $\mathcal{D}$. For simplicity, we concentrate on the case that the stationary point is rather close to the black hole horizon, in which $g$ is $O(1)$. In the case with a smaller $g$, which corresponds to a larger $\ell$, the deformation is expected to be even weaker.

When $g$ is $O(1)$, it is more convenient to use the parametrization $\lambda=\tilde{g} / g$ instead of $\tilde{g}$ introduced in Eqs. (35). Hence, our parametrization adopted here is

$$
e_{1}=a \epsilon, \quad e_{2}=1, \quad \gamma=g \epsilon^{2}, \quad \ell=\lambda \epsilon .
$$

The other parameters $m_{1}, m_{2}, \sigma_{1}^{2}$ and $\sigma_{2}^{2}$ are to be determined from Eqs. (13), (18) and (19) to the sufficiently high order in $\epsilon$. For the present purpose, the approximate expressions $(\underline{36}$ ) are not sufficient. 
To write down the metric and the electric potential in the $\left(\Delta, \theta_{1}\right)$ coordinates, we need to describe $r_{1}, r_{2}$ and $\theta_{2}$ in terms of $\left(\Delta, \theta_{1}\right)$. From the definitions (12) and (17), setting $z_{1}=0$ and $z_{2}=\ell$, we obtain

$$
\begin{aligned}
r_{2} & =m_{2}+\frac{\left(r_{1}-m_{1}\right) \cos \theta_{1}-\ell}{\cos \theta_{2}}, \\
\sin ^{2} \theta_{2} & =\frac{\left[\left(r_{1}-m_{1}\right)^{2}-\sigma_{1}^{2}\right] \sin ^{2} \theta_{1}}{\left(r_{2}-m_{2}\right)^{2}-\sigma_{2}^{2}}, \\
r_{1} & =r_{2}^{-1}\left[\left(e_{1}-\gamma-\gamma \cos \theta_{2}\right)\left(e_{2}+\gamma-\gamma \cos \theta_{1}\right)+\mathcal{D}\right],
\end{aligned}
$$

which can be solved by iteration in the presented order, once we have appropriate initial values for $r_{1}$ and $\theta_{2}$. The location of the singularity is specified by $\mathcal{D}=0$, where the lapse function $g_{t t}=-H$ and the electric potential $\Phi$ diverge. This point is expected to be close to the critical spheroid, and hence we have $r_{1} \approx m_{1}$. Then, we also have $\theta_{2} \approx-\pi$ near the singularity. Using these crude estimates as the initial condition for the iteration, we can solve these equations to a sufficiently high order in $\epsilon$.

Since the coordinate $\Delta$ is not best suited for seeing how the DRN solution deviates from the single RN singularity, it is better to further replace the radial coordinate to a physically well-motivated one. One simple possibility is to adopt the inverse of the electric potential as the radial coordinate, i.e.

$$
R:=\frac{a \tilde{c} \epsilon^{2}}{\left(\Phi-\Phi_{0}\right)}
$$

where $a \epsilon$ is the charge of the particle and we will find that the factor $\tilde{c} \epsilon$ takes care of the change of the time coordinate,

$$
d T:=\tilde{c} \epsilon d t
$$

with $\tilde{c}$ being a constant of $O(1)$ to be determined later, once we notice that the combination $A_{t} d t(=-\Phi d t)$ is gauge invariant. Here, the new time coordinate $T$ appropriate to describe the charged particle as a perturbed RN geometry elapses slower than the time of an asymptotic observer $t$ by the red shift factor $\tilde{c} \epsilon$ since the particle is staying deep inside the gravitational potential of the black hole. Also, a constant $\Phi_{0}$ was introduced to adjust the zero point of the electric potential. $\Phi_{0}$ will be also determined later. To focus on the range where $e_{1} / R=a \epsilon / R=O(1)$, we mainly use the rescaled radial coordinate $\varrho=R / \epsilon$, assuming $\varrho=O(1)$. (Notice that the coordinate $\varrho$ is different from $\rho$ in the cylindrical Weyl coordinates, used to describe the DRN solution in Eqs. 10 and (11).)

Then, it is straightforward to expand the metric in these coordinates up to $O(\epsilon)$. By choosing

$$
\begin{aligned}
\tilde{c} & =\frac{\sqrt{a \lambda(a \lambda-g)}}{a}-\epsilon \frac{\lambda\left(g^{2}-2 a g \lambda+2 a^{2} \lambda^{2}\right)}{2 a \sqrt{a \lambda(a \lambda-g)}}+O\left(\epsilon^{2}\right), \\
\Phi_{0} & =1-\lambda \epsilon+\epsilon^{2} \frac{\lambda(2 a \lambda-g)}{2 a}+O\left(\epsilon^{3}\right),
\end{aligned}
$$

the resulting metric is expressed in the following form

$$
\begin{aligned}
g_{T T} & =-\tilde{f}(\varrho)\left[1-\epsilon \cos \theta_{1}\left(\frac{2 g \varrho}{\sqrt{a \lambda(a \lambda-g)}}\right)+O\left(\epsilon^{2}\right)\right], \\
g_{R R} & =\frac{1}{\tilde{f}(\varrho)}\left[1+\epsilon \cos \theta_{1}\left\{\frac{6\left(a^{2}+\varrho^{2}\right)}{a}-\frac{2 \varrho(6 a \lambda-5 g)}{\sqrt{a \lambda(a \lambda-g)}}\right\}+O\left(\epsilon^{2}\right)\right], \\
g_{i j} & =R^{2} \gamma_{i j}\left[1+2 \epsilon \cos \theta_{1}\left\{\frac{\lambda\left(a^{2}+\varrho^{2}\right)-2 \varrho \sqrt{a \lambda(a \lambda-g)}}{a \lambda}\right\}+O\left(\epsilon^{2}\right)\right], \\
g_{R \theta_{1}} & =\epsilon R^{2} \sin \theta_{1}\left(\frac{\varrho}{a}-\sqrt{\frac{a \lambda}{(a \lambda-g)}}\right)+O\left(\epsilon^{2}\right),
\end{aligned}
$$

where we have defined

$$
\tilde{f}=\frac{a^{2}}{\varrho^{2}}-\left(\frac{2 \sqrt{a \lambda(a \lambda-g)}}{\lambda}-\epsilon \frac{g(2 a \lambda-g)}{\sqrt{a \lambda(a \lambda-g)}}\right) \frac{1}{\varrho}+1=\frac{q^{2}}{R^{2}}-\frac{2 \mu}{R}+1 .
$$

The second equality gives the expressions of $q$ and $\mu$ in terms of $a, g, \lambda$ and $\epsilon$, which are consistent with $q=a \epsilon, \mu=c \epsilon$ in Eqs. (9) supplemented with the relations $\lambda g \equiv \tilde{g}=4 a+O(\epsilon)$ and $g=2 \sqrt{a^{2}-c^{2}}+O(\epsilon)$ in Eqs. (38). In the next leading 
order in $\epsilon$ the metric given in Eqs. (A6) has only dipole type perturbation at around $R=O(\epsilon)$. Perturbations belonging to higher multipoles are of $O\left(\epsilon^{2}\right)$ or higher.

The asymptotic behavior of perturbations for small $R$ is to be determined in line with the nature of a charged particle. For example, when an external dipole field is imposed, a charged particle will gain some induced dipole moment. However, the magnitude of the induced dipole moment will depend on the property of the particle to some extent. In this sense there remains arbitrariness in the most relevant choice of the boundary conditions for our charged particle. Instead of discussing this, we show that the effect of the induced dipole of $O(\epsilon)$ in the expressions da6 does not contribute to the total energy of the system at the level of our interest, $O\left(\epsilon^{2}\right)$.

The key observation is that it is at $R=O(\epsilon)$ where the dipole perturbation is $O(\epsilon)$. This dipole field is a superposition of the external field caused by the presence of a charged black hole and the induced dipole of the point particle. Hence, the latter amplitude will be also at most $O(\epsilon)$. The dimensionless perturbation caused by an object having a dipole moment $D$ is $O\left(D / R^{2}\right)$. Therefore the magnitude of the dipole moment is evaluated as $D=O\left(\epsilon^{3}\right)$. In the region $R \gg O(\epsilon)$, the configuration can be understood as a RN black hole solution perturbed by a charged particle and an electric dipole placed at the same place. In such a regime the effect due to the dipole is at most $O\left(\epsilon^{3}\right)$. On the other hand, in the vicinity of $R=0$ we can evaluate the energy due to the dipole from the expanded metric presented in Eqs. A6. The explicit expanded metric shows that the monopole part of the perturbation starts with $O\left(\epsilon^{2}\right)$. Since the change of the mass $\delta \mu$ will appear in the monopole part of the dimensionless metric perturbation as $\delta \mu / R$, we find that $\delta \mu$ is at most $O\left(\epsilon^{3}\right)$ in the region $R \lesssim \epsilon$. As a result, we conclude that the contribution of the dipole to the total energy is $O\left(\epsilon^{3}\right)$. The higher multipoles can be discussed in a similar way, and we find that they are even higher order in $\epsilon$.

\section{Appendix B: $\epsilon$-expansion of the parameters in the DRN solution}

This appendix is devoted to deriving the appropriate order of $\gamma$ and $\ell$ in terms of $\epsilon$-parametrization. Our starting point is the assignment of the ordering that defines the setup of our problem given in Eqs. (34). The parameters are also constrained by the inequalities $\sigma_{1}^{2}<0$ and $\sigma_{2}^{2}>0$, which are required because $\sigma^{2}$ is positive for a $\mathrm{BH}$ and negative for a singularity.

From the ordering given in Eqs. (34), the leading term of $\gamma$ in Eq. 18) can be read as

$$
\gamma=\frac{e_{1}-m_{1}}{1+\ell}(1+O(\epsilon))
$$

Substituting the above approximate $\gamma$ into the balance condition [19], we find

$$
\ell\left(e_{1}-m_{1}\right)=O\left(\epsilon^{2}\right) .
$$

Multiplying $\ell$ on both sides of Eq. (B1), with the aid of (B2) we have $\ell \gamma=O\left(\epsilon^{2}\right)$. Next, using the last equality in Eqs. (34), we find

$$
e_{1}-m_{1}=\varpi+O\left(\epsilon^{2}\right) .
$$

Plugging Eq. (B3) into Eq. (B 2$)$, we also have $\ell \varpi=O\left(\epsilon^{2}\right)$.

We now show that Eq. $\mathrm{B2}$ is in fact further suppressed. Substituting Eq. (18) into Eq. 19) and expanding it up to $O\left(\epsilon^{2}\right)$ after multiplication of the factor $\left(\ell+m_{1}+m_{2}\right)$ on both sides, and using Eq. (B-2) and the relation $\ell \varpi=O\left(\epsilon^{2}\right)$, we obtain $2 m_{1}\left(e_{1}-m_{1}-\varpi\right)+\ell\left(e_{1}-m_{1}\right)=O\left(\epsilon^{3}\right)$. With Eq. $[\mathbf{B} 3$, this reduces to

$$
\ell\left(e_{1}-m_{1}\right)=O\left(\epsilon^{3}\right),
$$

which also implies $\ell \gamma=O\left(\epsilon^{3}\right)$ in the same manner as above and $\ell \varpi=O\left(\max \left(\epsilon^{3}, \ell \epsilon^{2}\right)\right)$ with the aid of Eq. (B3). From Eq. (18),

$$
m_{1}=\frac{(1+\varpi)\left(e_{1}-\gamma\right)-\ell \gamma}{1+\gamma},
$$

follows. Substituting this expression for $m_{1}$ into $\sigma_{1}^{2}$ given in Eqs. (13), with the aid of the relations (B1) and (B3), we obtain

$$
\sigma_{1}^{2}=\gamma^{2}+O\left(\epsilon^{4}\right)
$$

Thus, the requirement that $\sigma_{1}^{2}<0$ can be consistent only when $\gamma=O\left(\epsilon^{2}\right)$. Then, immediately both $e_{1}-m_{1}$ and $\varpi$ turn out to be $O\left(\epsilon^{2}\right)$

To summarize, if $\ell$ is $O(\epsilon)$, we find that both $e_{1}-m_{1}=\gamma+O\left(\epsilon^{3}\right)$ and $\varpi$ are $O\left(\epsilon^{2}\right)$. When $\ell$ is not as small as $O(\epsilon)$, the order of $e_{1}-m_{1}$ and $\gamma$ depends on the magnitude of $\ell$ in such a way that $\ell\left(e_{1}-m_{1}\right)=\ell(\ell+1) \gamma+O\left(\epsilon^{4}\right)$ becomes $O\left(\epsilon^{3}\right)$. By contrast, $\varpi$ stays $O\left(\epsilon^{2}\right)$ irrespective of the magnitude of $\ell$. 
Appendix C: Mapping an exact static solution to an equilibrium configuration of a charged particle in the RN spacetime

In this appendix we consider the mapping of the DRN static solution to an equilibrium configuration of a charged particle in a RN black hole background: the BHP picture. It is not so trivial to find the relations between the different descriptions, especially the relations between $\tilde{g}$ and $g$ in Eqs. (38), and the parameters $a, b$ and $c$ given in Eqs. (9). To obtain these relations, we focus on two geometrical quantities: one is the proper distance $L$ along the symmetric axis between the event horizon of the black hole and the equilibrium position of the particle, and the other is the proper distance $D$ from the event horizon to the asymptotic region, measured along the symmetric axis in the direction opposite to the particle. For our current purpose, in linear perturbation theory analysis, it is enough to estimate the emitted energy at the leading order in $\epsilon$ since the energy flux (52) is always $O\left(\epsilon^{2}\right)$. The back reaction effects on the orbit contribute only to the higher order corrections in the energy flux. Therefore we consider the mapping at the level of linear perturbation of a RN black hole, restricted to the order in $\epsilon$ expansion necessary to specify the setup in the BHP picture.

We first compute the above geometrical quantities in the BHP picture. Since we consider the equilibrium state, however, the parameters $a, b$ and $c$ in Eqs. (9) are not independent but satisfy the relation

$$
b=\sqrt{a^{2}-c^{2}}+O(\epsilon),
$$

which is derived from the equilibrium conditions $d R / d s=d R^{2} / d s^{2}=0$. The value of the radial coordinate at the equilibrium position is also derived from these equilibrium conditions as

$$
r_{0}=1+\frac{2 a}{\sqrt{a^{2}-c^{2}}} \epsilon+O\left(\epsilon^{2}\right) .
$$

Then, the quantities mentioned above are calculated in the BHP picture as

$$
\begin{aligned}
L & =\int_{r_{+}}^{r_{0}} \sqrt{g_{r r}} d r^{\prime}=\int_{r_{+}}^{r_{0}} \frac{d r^{\prime}}{\sqrt{f\left(r^{\prime}\right)}}=\frac{1}{2} \log \frac{a+c}{a-c}+O(\epsilon), \\
D(r) & =\int_{r_{+}}^{r} \sqrt{g_{r r}} d r^{\prime}=\int_{r_{+}}^{r} \frac{d r^{\prime}}{\sqrt{f\left(r^{\prime}\right)}}=-\log (\epsilon)-1-(r+\log r)+O\left(\frac{1}{r}, \epsilon\right),
\end{aligned}
$$

where $f(r)$ is the metric function of the RN black hole defined by Eq. (3).

Next we evaluate $L$ and $D$ in the DRN solutions. In the DRN solution (10)-(18), we can choose $\left(z_{1}, z_{2}\right)=(-l, 0)$ without loss of generality. For this choice, the event horizon on the axis is at $(\rho, z)=\left(0, \pm \sigma_{2}\right)$. Then, the proper distance $L$ in the DRN solution is formally given by

$$
L=\int_{-\ell}^{-\sigma_{2}} \sqrt{F(0, z)} d z
$$

where $F(\rho, z)$ is one of the metric functions of the DRN solution defined by Eq. (15) and we take the upper end of the integral at $z=-\ell$, which is sufficiently close to the singularity of the body 1 . In the range of $-\ell<z<-\sigma_{2}$ on the symmetric axis, from Eqs. [12, we obtain

$$
\left\{\begin{array}{l}
r_{1}=z+m_{1}+\ell, \quad \theta_{1}=0 \\
r_{2}=-z+m_{2}, \quad \theta_{2}=\pi
\end{array}\right.
$$

and then the metric function on the axis is given by

$$
F(0, z)=\frac{\left[\left(-z+m_{2}\right)\left(z+m_{1}+\ell\right)-e_{1} e_{2}\right]^{2}}{\left[(z+\ell)^{2}-\sigma_{1}^{2}\right]\left[z^{2}-\sigma_{2}^{2}\right]} .
$$

The direct integration of Eq. (C5) gives a complicated combination of incomplete elliptical integrals that is inconvenient for our analysis. To avoid the complication, we divide the range of the integral $\left[-\ell,-\sigma_{2}\right]$ into $\left[-\ell,-\ell+A \epsilon^{3 / 2}\right]$ and $\left[-\ell+A \epsilon^{3 / 2},-\sigma_{2}\right]$, where $A$ is an arbitrary constant of $O(1)$. In the former range, we expand the term $1 / \sqrt{z^{2}-\sigma_{2}^{2}}$ in the integrand of Eq. (C5) with respect to $z+\ell=O\left(\epsilon^{3 / 2}\right)\left(\ll \sigma_{2}\right)$, keeping the term $1 / \sqrt{(z+\ell)^{2}-\sigma_{1}^{2}}$. On the other hand, in the latter region, we expand $1 / \sqrt{(z+\ell)^{2}-\sigma_{1}^{2}}$ with respect to $\sigma_{1}$ while the factor $1 / \sqrt{z^{2}-\sigma_{2}^{2}}$ is kept unexpanded. Then, one can perform the integrals to obtain an approximate estimate of $L$ as

$$
L=\left(\int_{-\ell}^{-\ell+A \epsilon^{3 / 2}}+\int_{-\ell+A \epsilon^{3 / 2}}^{-\sigma_{2}}\right) \sqrt{F(0, z)} d z=\operatorname{arccosh} \sqrt{\frac{a \tilde{g}}{g^{2}}}+O(\epsilon) .
$$


The proper distance $D$ from the event horizon of the black hole to the asymptotic region $z \gg 1$ along the symmetric axis is also formally given by

$$
D=\lim _{z \rightarrow \infty} \int_{\sigma_{2}}^{z} \sqrt{F\left(0, z^{\prime}\right)} d z^{\prime} .
$$

Here it should be noticed that, unlike the case of $L$, we take the lower end of the integral at $z=\sigma_{2}$ because the integral is performed in the direction opposite to the body 1 (singularity). In the range of the integral on the right hand side of Eq. (C9), the bipolar coordinates are given by

$$
\left\{\begin{array}{l}
r_{1}=z+m_{1}+\ell, \quad \theta_{1}=0 \\
r_{2}=z+m_{2}, \quad \theta_{2}=0
\end{array}\right.
$$

Substituting Eqs. (C10) into Eq. [15], the metric function is rewritten as

$$
F(0, z)=\frac{\left[\left(z+m_{2}\right)\left(z+m_{1}+\ell\right)-\left(e_{1}-2 \gamma\right) e_{2}\right]^{2}}{\left[(z+\ell)^{2}-\sigma_{1}^{2}\right]\left[z^{2}-\sigma_{2}^{2}\right]}=\frac{\left(z+m_{2}\right)^{2}}{z^{2}-\sigma_{2}^{2}}(1+O(\epsilon)) .
$$

Then, the integral (C9) with Eq. (C11) can be evaluated. Since the coordinate $z$ is related to $r$ on the axis as $z=r-1+O(\epsilon)$, which can be easily verified by looking at, say, the lapse function, we finally obtain

$$
D=-\log \left(\frac{\epsilon}{2} \sqrt{\frac{\tilde{g}}{a}}\right)-1+(r+\log r)+O\left(\frac{1}{r}, \epsilon\right) .
$$

Comparing Eq. (C4) to Eq. (C12) and Eq. (C3) to Eq. (C8), we establish relations between the two sets of parameters presented in Eqs. (38), and with the aid of the constraint equation (C1) valid up to $O(\epsilon)$ we obtain $b$.

Appendix D: Evaluate the energy radiated to infinity from a moving charged particle

In this appendix we evaluate the energy $E_{\infty}$ emitted to infinity from a charged particle that falls into a RN black hole along a radial orbit.

\section{Energy flux formula}

First, we derive the effective energy flux generated from the scalar-type perturbation in the Kodama-Ishibashi (KI) formalism. The energy flux carried by gravitational waves and that by electromagnetic waves are decoupled in the asymptotic region $r \rightarrow$ $+\infty$, and they are, respectively, given in terms of the perturbation of the metric $h_{\mu \nu}$ and that of the electro-magnetic field strength $f_{\mu \nu}$ as [21]

$$
\dot{E}_{\infty}^{\mathrm{GW}}:=-r^{2} \int \frac{d \Omega}{4 \kappa} \frac{\partial h_{\alpha \beta}}{\partial t} \frac{\partial h^{\alpha \beta}}{\partial r}, \quad \dot{E}_{\infty}^{\mathrm{EM}}=-r^{2} \int d \Omega f_{t \rho} f_{r}^{\rho},
$$

in the transverse-traceless gauge defined by $h_{\rho}^{\rho}=0, \nabla^{\nu} h_{\mu \nu}=0$. Here, averaging over several wavelengths of radiation is assumed. We rewrite these expressions (D1) in terms of the master variables $\Phi_{ \pm}$in the KI formalism. To do so, as an intermediate step we consider $\Phi$ and $\mathcal{A}$ that are related to $\Phi_{ \pm}$at the level of coefficients of the spherical harmonics expansion by

$$
\Phi_{ \pm}=a_{ \pm} \Phi+b_{ \pm} \mathcal{A}
$$

with the coefficients defined in Eqs. 45, where the indices of spherical harmonics, $\mathfrak{l}$ and $\mathfrak{m}$, are suppressed for notational simplicity. At the leading order in the limit $r \rightarrow+\infty$, the master variables $\Phi$ and $\mathcal{A}$ are, respectively, related to the perturbations of gravitational and electro-magnetic fields as

$$
h_{i j}:=\sum_{\mathfrak{l}, \mathfrak{m}} \mathfrak{l}(\mathfrak{l}+1) r \Phi \mathbb{S}_{i j}, \quad f_{a i} \approx \sum_{\mathfrak{l}, \mathfrak{m}} \sqrt{\mathfrak{l}(\mathfrak{l}+1)} \epsilon_{a b}\left(D^{b} \mathcal{A}\right) \mathbb{S}_{i},
$$

where $a, b$-indices run over $t, r$-coordinates, while $i, j$-indices over angular coordinates. The other components are of higher order in $1 / r$. The totally anti-symmetric symbol $\epsilon_{a b}$ is defined so that $\epsilon_{t r}=1$. The harmonics are defined as

$$
\mathbb{S}:=Y_{\mathfrak{l m}}, \quad \mathbb{S}_{i}:=-\frac{1}{\sqrt{\mathfrak{l}(\mathfrak{l}+1)}} \hat{D}_{i} Y_{\mathfrak{l m}}, \quad \mathbb{S}_{i j}:=\frac{1}{\mathfrak{l}(\mathfrak{l}+1)} \hat{D}_{i} \hat{D}_{j} Y_{\mathfrak{l m}}+\frac{1}{2} \gamma_{i j} Y_{\mathfrak{l m}},
$$


where $Y_{\operatorname{lm}}$ are the usual spherical harmonics on a unit sphere $S^{2}$ and $\hat{D}_{i}$ represents covariant differentiation with respect to the metric of $S^{2}, \gamma_{i j}$.

Then, substituting Eqs. (D2), (D3) and (D4) into Eq. (D1), the total energy flux escaping to infinity is evaluated as

$$
\begin{aligned}
\dot{E}_{\infty} & =-\sum_{\mathfrak{l}, \mathfrak{m}} 4 \pi \mathfrak{l}(\mathfrak{l}+1)\left(\frac{\partial \mathcal{A}}{\partial t} \frac{\partial \overline{\mathcal{A}}}{\partial r}+\frac{(\mathfrak{l}-1)(\mathfrak{l}+2)}{8 \kappa^{2}} \frac{\partial \Phi}{\partial t} \frac{\partial \bar{\Phi}}{\partial r}\right) \\
& =-\sum_{\mathfrak{l}, \mathfrak{m}} \frac{4 \pi \mathfrak{l}(\mathfrak{l}+1)}{9 \kappa^{2} \nu(M+\nu)}\left(\frac{\partial \Phi_{+}}{\partial t} \frac{\partial \bar{\Phi}_{+}}{\partial r}+\frac{(\mathfrak{l}-1)(\mathfrak{l}+2)}{16} \frac{\partial \Phi_{-}}{\partial t} \frac{\partial \bar{\Phi}_{-}}{\partial r}\right),
\end{aligned}
$$

where quantities with " - " represent the complex conjugations, and all terms suppressed by $1 / r$ are neglected. Note that this expression is valid for all modes including the exceptional mode with $\mathfrak{l}=1$. However, for the exceptional mode the second term in the parentheses on the last line vanishes. Hence, we find that $\Phi_{-}$is irrelevant for this mode, which is consistent with the fact that physical degrees of freedom for the metric perturbation are absent for the exceptional mode. (See Ref. [12] for further details.)

We would like to add a short remark about the expression for the energy flux (D5). This is a special case of a more general expression for the energy flux defined as an integration over an arbitrary 2-surface of constant $r$ :

$$
\dot{E}(r)=-\sum_{\mathfrak{l}, \mathfrak{m}} \frac{4 \pi \mathfrak{l}(\mathfrak{l}+1) f}{9 \kappa^{2} \nu(M+\nu)}\left(\frac{\partial \Phi_{+}}{\partial t} \frac{\partial \bar{\Phi}_{+}}{\partial r}+\frac{(\mathfrak{l}-1)(\mathfrak{l}+2)}{16} \frac{\partial \Phi_{-}}{\partial t} \frac{\partial \bar{\Phi}_{-}}{\partial r}\right),
$$

which is identical to Eq. (D5) in the limit $r \rightarrow \infty$. Here, $f$ is the metric function of the background RN black hole given in Eq. (3). To verify that the above expression is conserved in the absence of source terms, we focus on the fact that the master equation (40) is derived from the variational principle of the action

$$
S:=\int d^{2} x \sqrt{-g_{(2)}}\left(-\frac{1}{2} g_{(2)}^{a b} \nabla_{a}^{(2)} \Phi_{ \pm} \nabla_{b}^{(2)} \Phi_{ \pm}-V_{ \pm} \Phi_{ \pm}^{2}\right),
$$

where $g_{(2)}^{a b}$ is the two dimensional metric composed of the $(t, r)$ components of the RN metric, $\nabla_{a}^{(2)}$ is the covariant differentiation with respect to $g_{(2)}^{a b}$ and $g_{(2)}$ is the determinant of this two dimensional metric. The $t$ and $r$ components are labeled by the Latin indices, $a, b$. Once the action (D7) is at hand, its variation with respect to $g_{(2)}^{a b}$ gives a symmetric tensor $T_{a b}$ that satisfies the conservation law $\nabla_{b}^{(2)} T_{a}{ }^{b}=0$. Then, one can construct a conserved current $T_{a b}\left(\partial_{t}\right)^{b}$ associated with the background Killing field $\left(\partial_{t}\right)$. Thus, we find the conserved flux per unit coordinate time is proportional to

$$
T_{a b} n^{a}\left(\partial_{t}\right)^{b} \sqrt{g_{t t}^{(2)}} \propto f \frac{\partial \Phi_{ \pm}}{\partial t} \frac{\partial \Phi_{ \pm}}{\partial r}
$$

where $n_{a}$ is the unit vector normal to a surface of constant $r$. Since the expression (D6) is a linear combination of these conserved fluxes, it is guaranteed to be conserved. For any wave packet the time integral of this flux is independent of $r$, and agrees with the net energy flux evaluated at infinity. The net energy flux is also a quantity that must be independent of $r$. Therefore for any wave packet the time integral of this flux (D6) evaluated at any radius gives the net energy flux, and we conclude that Eq. (D6) is indeed an expression for the conserved energy flux valid for any value of $r$.

\section{Evaluation of the energy flux from a radially falling particle}

Now we turn to the evaluation of the coefficients $X_{ \pm}(\omega)$ in Eq. (51). The explicit form of these coefficients are, with $Y_{\mathfrak{l} 0}:=$ $\sqrt{(2 \mathfrak{l}+1) / 4 \pi}$, expressed as

$$
\begin{aligned}
X_{ \pm}(\omega) / Y_{\mathfrak{l} 0}= & i \frac{8 q b_{ \pm}}{\mathfrak{l}(\mathfrak{l}+1) \omega} \int_{r_{+}}^{r_{0}}\left[\frac{z f}{r^{2} H} \Phi_{ \pm}^{\mathrm{in}} e^{i \omega T}\right] d r \\
& -i \frac{2 \sqrt{2} \kappa \mu Q b_{ \pm}}{\omega} \int_{r_{+}}^{r_{0}}\left[\frac{1}{r^{3} H}\left(f \frac{d T}{d s}\right) \Phi_{ \pm}^{\mathrm{in}} e^{i \omega T}\right] d r \\
& -i \frac{q b_{ \pm}}{\mathfrak{l}(\mathfrak{l}+1) \omega} \int_{r_{+}}^{r_{0}}\left[\frac{V_{ \pm}}{f} \Phi_{ \pm}^{\mathrm{in}} e^{i \omega T}\right] d r \\
& +i \frac{\sqrt{2} \kappa q Q}{\mathfrak{l}(\mathfrak{l}+1) \omega} \int_{r_{+}}^{r_{0}}\left[\frac{P_{S 1}}{r^{3} H^{2}} a_{ \pm} \Phi_{ \pm}^{\mathrm{in}} e^{i \omega T}\right] d r
\end{aligned}
$$




$$
\begin{aligned}
& -i \frac{2 \sqrt{2} \kappa q Q}{\omega} \int_{r_{+}}^{r_{0}}\left[\frac{1}{r^{3} H} a_{ \pm} \Phi_{ \pm}^{\mathrm{in}} e^{i \omega T}\right] d r \\
& +i \frac{\kappa^{2} \mu}{\omega} \int_{r_{+}}^{r_{0}}\left[\frac{P_{S 3}}{r^{2} H^{2}}\left(f \frac{d T}{d s}\right) a_{ \pm} \Phi_{ \pm}^{\mathrm{in}} e^{i \omega T}\right] d r \\
& +2 \kappa^{2} \mu \int_{r_{+}}^{r_{0}}\left[\frac{1}{r f H}\left(\frac{d R}{d s}\right) a_{ \pm} \Phi_{ \pm}^{\mathrm{in}} e^{i \omega T}\right] d r \\
& -i \frac{2 \kappa^{2} \mu}{\omega} \int_{r_{+}}^{r_{0}}\left[\frac{1}{r^{2}} \frac{d}{d r}\left(\frac{a_{ \pm} r}{H} \Phi_{ \pm}^{\mathrm{in}}\right)\left(f \frac{d T}{d s}\right) e^{i \omega T}\right] d r
\end{aligned}
$$

for both generic and exceptional modes. In the above expression, $V_{ \pm}$are the effective potentials of the scalar-type perturbation defined in Eq. (41), and the functions $H, P_{S 1}$ and $P_{S 3}$ are as defined in Eqs. (48). The components $(t, r)=(T(s), R(s))$ specifies the trajectory of a charged particle obtained by integrating Eqs. (4) and (5) with the initial condition that the particle is at rest at the equilibrium position $r=r_{0}$ in the limit $t \rightarrow-\infty$.

Notice that the integrands in the above expression do not possess any divergences at $r=r_{0}$. By contrast, in the limit $r=r_{+}$ the integrands of the last two terms in Eq. (D9) diverge. To see this, recall that $f$ vanishes at $r=r_{+}$and $d T / d s$ and $d \Phi_{ \pm}^{\text {in }} / d r$ behave like $\propto f^{-1}$ there, while the other functions including $d R / d s$ are regular. To ameliorate this singular behavior, we perform the integration by parts in the following manner. First, we rewrite $e^{i \omega T}$ in the second to last term by using the following identity

$$
e^{i \omega T}=\frac{1}{i \omega} \frac{d R}{d T}\left(\frac{d e^{i \omega T}}{d r}\right)
$$

Then, using the relation $f(d T / d s)^{2}-f^{-1}(d R / d s)^{2}=1$, which is simply the normalization condition of the four velocity, the contribution from the last two terms can be rewritten as

( the last two terms in Eq. (D9) )

$$
\begin{aligned}
& =\frac{2 \kappa^{2} \mu}{i \omega} \int_{r_{+}}^{r_{0}}\left[\frac{a_{ \pm}}{r H} \frac{d T}{d s} \Phi_{ \pm}^{\text {in }}\left\{f-\left(\frac{d T}{d s}\right)^{-2}\right\}\left(\frac{d e^{i \omega T}}{d r}\right)+\frac{f}{r^{2}} \frac{d T}{d s} e^{i \omega T} \frac{d}{d r}\left(\frac{a_{ \pm} r}{H} \Phi_{ \pm}^{\text {in }}\right)\right] d r \\
& =\frac{2 \kappa^{2} \mu}{i \omega} \int_{r_{+}}^{r_{0}}\left[\frac{f}{r^{2}} \frac{d T}{d s} \frac{d}{d r}\left(\frac{a_{ \pm} r}{H} \Phi_{ \pm}^{\text {in }} e^{i \omega T}\right)-\frac{a_{ \pm}}{r H}\left(\frac{d T}{d s}\right)^{-1} \Phi_{ \pm}^{\text {in }}\left(\frac{d e^{i \omega T}}{d r}\right)\right] d r \\
& =-\frac{2 \kappa^{2} \mu}{i \omega} \int_{r_{+}}^{r_{0}}\left[\frac{a_{ \pm} r}{H} \Phi_{ \pm}^{\text {in }} \frac{d}{d r}\left(\frac{f}{r^{2}} \frac{d T}{d s}\right)-\frac{d}{d r}\left\{\frac{a_{ \pm} f}{r H}\left(f \frac{d T}{d s}\right)^{-1} \Phi_{ \pm}^{\text {in }}\right\}\right] e^{i \omega T} d r,
\end{aligned}
$$

where we performed integration by parts in the last equality and dropped the surface terms. The surface terms take the form of an infinitely oscillating function such as $e^{i \omega T}$ multiplied by a regular factor near the boundaries. Such terms can be safely neglected by introducing an infinitesimal damping factor as usual. In the final expression the integrand is free from divergence on both boundaries at $r=r_{+}$and $r_{0}$.

[1] R. Penrose, Phys. Rev. Lett. 14, 57 (1965).

[2] R. M. Wald, arXiv:gr-qc/9710068

[3] P. O. Mazur, J. Phys. A 15, 3173 (1982).

[4] R. M. Wald, Ann. Phys. 82, 548 (1974).

[5] J. M. Cohen and R. Gautreau, Phys. Rev. D 19, 2273 (1979); T. Needham, Phys. Rev. D 22, 791 (1980); I. Semiz, Class. Quant. Grav. 7, 353 (1990); J. D. Bekenstein and C. Rosenzweig, Phys. Rev. D 50, 7239 (1994) [arXiv:gr-qc/9406024]; I. Semiz, Gen. Rel. Grav. 43, 833 (2011) [arXiv:gr-qc/0508011].

[6] F. de Felice and Y. Q. Yu, Class. Quant. Grav. 18, 1235 (2001).

[7] V. E. Hubeny, Phys. Rev. D 59, 064013 (1999) [arXiv:gr-qc/9808043].

[8] T. Jacobson and T. P. Sotiriou, Phys. Rev. Lett. 103, 141101 (2009) [Erratum-ibid. 103, 209903 (2009)] [arXiv:0907.4146 [gr-qc]].

[9] A. Saa and R. Santarelli, Phys. Rev. D 84, 027501 (2011) |arXiv:1105.3950 [gr-qc]].

[10] S. Hod, arXiv:gr-qc/9908004, S. Hod and T. Piran, Gen. Rel. Grav. 32, 2333 (2000) [arXiv:gr-qc/0011003]; S. Hod, Phys. Rev. D 66, 024016 (2002) |arXiv:gr-qc/0205005].

[11] M. Kesden, G. Lockhart and E. S. Phinney, Phys. Rev. D 82, 124045 (2010) [arXiv:1005.0627 [gr-qc]]; E. Barausse, V. Cardoso and G. Khanna, Phys. Rev. Lett. 105, 261102 (2010) arXiv:1008.5159 [gr-qc]]; E. Barausse, V. Cardoso and G. Khanna, arXiv:1106.1692 [gr-qc].

[12] H. Kodama and A. Ishibashi, Prog. Theor. Phys. 111, 29 (2004) arXiv:hep-th/0308128 ; A. Ishibashi and H. Kodama, arXiv:1103.6148 [hep-th]. 
[13] M. Johnston, R. Ruffini and F. Zerilli, Phys. Rev. Lett. 31 (1973) 1317; M. Johnston, R. Ruffini and F. Zerilli, Phys. Lett. B 49 (1974) 185.

[14] G. A. Alekseev and V. A. Belinski, Phys. Rev. D 76, 021501 (2007) arXiv:0706.1981 [gr-qc]]; G. A. Alekseev and V. A. Belinski, arXiv:0710.2515 [gr-qc]; G. A. Alekseev and V. A. Belinski, arXiv:1103.0582 [gr-qc].

[15] V. S. Manko, Phys. Rev. D 76, 124032 (2007) [arXiv:0710.2158 [gr-qc]].

[16] D. Bini, A. Geralico and R. Ruffini, Phys. Rev. D 75, 044012 (2007) [arXiv:gr-qc/0609041].

[17] S. D. Majumdar, Phys. Rev. 72, 390 (1947); A. Papapetrou, Proc. R. Irish Acad. 51, 191 (1947).

[18] F. J. Zerilli, Phys. Rev. D 9, 860 (1974); V. Moncrief, Phys. Rev. D 12, 1526 (1975); S. Chandrasekhar, "The mathematical theory of black holes," Oxford, UK: Clarendon (1992) 646 p.

[19] Y. Mino, M. Sasaki and T. Tanaka, Phys. Rev. D 55, 3457 (1997) [arXiv:gr-qc/9606018]; T. C. Quinn and R. M. Wald, Phys. Rev. D 56, 3381 (1997) |arXiv:gr-qc/9610053].

[20] T. Futamase, P. A. Hogan and Y. Itoh, Phys. Rev. D 78, 104014 (2008) arXiv:0811.4020 [gr-qc]].

[21] C. W. Misner, K. S. Thorne and J. A. Wheeler, “Gravitation,” San Francisco 1973, 1279p 